\title{
A LIOUVILLE-TYPE THEOREM ON HALF-SPACES FOR SUB-LAPLACIANS
}

\author{
ALESSIA E. KOGOJ
}

(Communicated by Jeremy Tyson)

\begin{abstract}
Let $\mathcal{L}$ be a sub-Laplacian on $\mathcal{L}^{N}$ and let $\mathbb{G}=\left(\mathcal{L}^{N}, \circ, \delta_{\lambda}\right)$ be its related homogeneous Lie group. Let $\mathbb{E}$ be a Euclidean subgroup of $\mathcal{L}^{N}$ such that the orthonormal projection $\pi: \mathbb{G} \longrightarrow \mathbb{E}$ is a homomorphism of homogeneous groups, and let $\langle$,$\rangle be an inner product in \mathbb{E}$. Given $\alpha \in \mathbb{E}, \alpha \neq 0$, define $\Omega(\alpha):=\{x \in \mathbb{G}:\langle\alpha, \pi(x)\rangle>0\}$. We prove the following Liouville-type theorem.

If $u$ is a nonnegative $\mathcal{L}$-superharmonic function in $\Omega(\alpha)$ such that $u \in$ $L^{1}(\Omega(\alpha))$, then $u \equiv 0$ in $\Omega(\alpha)$.
\end{abstract}

\section{INTRODUCTION}

In [14] F. Uguzzoni proved the following Liouville-type theorem.

Theorem A. Let $\Delta_{\mathbb{H}_{n}}$ be a sub-Laplacian on the Heisenberg group $\mathbb{H}_{n}$ and let $\Omega$ be a half-space of $\mathbb{H}_{n}$ whose boundary is parallel to the center of $\mathbb{H}_{n}$. If $u$ is a nonnegative $\Delta_{\mathbb{H}_{n}}$-superharmonic function such that $u \in L^{1}(\Omega)$, then $u \equiv 0$.

The aim of this note is to show that an analogous result holds in the general setting of the sub-Laplacians on $\mathbb{R}^{N}$.

Let $\mathcal{L}$ be a sub-Laplacian in $\mathbb{R}^{N}$ whose related homogeneous Lie group is $\left(\mathbb{G}, \circ, \delta_{\lambda}\right)$. Let $\mathbb{E}$ be an Euclidean subgroup of $\mathbb{R}^{N}$ such that the orthonormal projection

$$
\pi: \mathbb{G} \longrightarrow \mathbb{E}
$$

is a homomorphism of homogeneous Lie groups, i.e.,

$$
\pi\left(x \circ y^{-1}\right)=\pi(x)-\pi(y), \quad \pi\left(\delta_{\lambda}(x)\right)=\lambda \pi(x),
$$

for every $x, y \in \mathbb{G}$ and every $\lambda>0$.

Let $\langle$,$\rangle be an inner product in \mathbb{E}$ and, for every $\alpha \in \mathbb{E}, \alpha \neq 0$, define

$$
\Omega(\alpha):=\{x \in \mathbb{G}:\langle\alpha, \pi(x)\rangle>0\} .
$$

The main result of this paper is the following Liouville-type theorem.

Theorem 1.1. Let $u: \Omega(\alpha) \longrightarrow]-\infty, \infty]$ be a $\mathcal{L}$-superharmonic function in $\Omega(\alpha)$. If $u \geq 0$ and $u \in L^{1}(\Omega(\alpha))$, then

$$
u \equiv 0 \text { in } \Omega(\alpha)
$$
2013.

Received by the editors September 11, 2012 and, in revised form, March 10, 2013 and March 24,

2010 Mathematics Subject Classification. Primary 35B53, 35R03, 31C05, 31B05; Secondary $35 \mathrm{H} 20,35 \mathrm{H} 10$.

Key words and phrases. Liouville-type theorems, half-spaces, superharmonic functions, subLaplacians, homogeneous Lie groups. 
Liouville-type theorems in half-spaces for sub-Laplacian play a crucial role in looking for solutions to semilinear boundary value problems; see, e.g., [2, [1, 3], [7. Liouville-type theorems in the whole space in a sub-Riemannian setting have received increasing attention in recent years; see, e.g., [4] (Section 5.8), 10], 11, [12], 13], the references therein, and the recent deep papers by D'Ambrosio and Mitidieri both for Riemannian and sub-Riemannian results ([8], 9]).

We would like to stress that to prove Theorem 1.1 we exploit a technique which is different with respect to the one used in the previous quoted papers. We follow the approach of Uguzzoni in [14 based on suitable mean value operators on the level set of the fundamental solution of $\mathcal{L}$ and, moreover, a kind of invariance of $\Omega(\alpha)$ with respect to suitable left translations of $\mathbb{G}$. For this last reason our method cannot work for half-spaces without this invariance property.

We would also like to stress that our result, in the case of the Heisenberg group $\mathbb{H}_{n}$, gives back the result of Uguzzoni. As already noticed in [14, the assumption $u \in L^{1}(\Omega(\alpha))$ cannot be improved in the following sense.

Proposition 1.2. Let $p \in] 1,+\infty[$ be fixed, and let $\mathbb{G}$ be a Lie group whose homogeneous dimension $Q$ satisfies

$$
\frac{Q}{2}>\frac{p}{p-1}
$$

Then for every $\alpha \in \mathbb{E}$ there exists a strictly positive $\Delta_{\mathbb{G}}$-harmonic function $u$ in $\Omega(\alpha)$ such that

$$
\int_{\Omega(\alpha)} u^{p} d x<+\infty
$$

In particular this statement holds for the classical Laplacian $\Delta$ in $\mathbb{R}^{N}$ if $\frac{N}{2}>\frac{p}{p-1}$.

In Remark 3.1 we will recognize also that the assumption $u \geq 0$ cannot be removed from Theorem 1.1

We close this introduction by showing some explicit examples of applications of our Theorem 1.1 .

Example 1.3. In $\mathbb{R}^{N}=\mathbb{R}^{m} \times \mathbb{R}^{n}$, whose point is denoted by $(x, t), x \in \mathbb{R}^{m}, t \in \mathbb{R}^{n}$, consider the linear second order partial differential operator (PDO)

$$
\mathcal{L}=\Delta_{x}+\frac{1}{4}|x|^{2} \Delta_{t}+\sum_{k=1}^{n}\left\langle B^{(k)} x, \nabla_{x}\right\rangle \partial_{t_{k}}
$$

where $\Delta_{x}=\sum_{j=1}^{m} \partial_{x_{j}}^{2}$ and $\Delta_{t}=\sum_{j=1}^{n} \partial_{t_{j}}^{2}$ are the usual Laplace operator in $\mathbb{R}^{m}$ and in $\mathbb{R}^{n}$, respectively. $\nabla_{x}=\left(\partial_{x_{1}}, \ldots, \partial_{x_{m}}\right)$ and $B^{(1)}, \ldots, B^{(m)}$ are $m \times m$ matrices having the following properties:

(i) $B^{(k)}$ is skew-symmetric and orthogonal, $k=1, \ldots, m$;

(ii) $B^{(i)} B^{(j)}=-B^{(j)} B^{(i)}$ for every $i, j \in\{j=1, \ldots, m\}, i \neq j$.

Then $\mathcal{L}$ in (3.1) is a sub-Laplacian on a group of Heisenberg type $\mathbb{H}$, and the map $\pi: \mathbb{H} \longrightarrow \mathbb{R}^{m}, \pi(x, t)=x$ is a homomorphism of homogeneous groups (see 6 , Section 3.6]).

For every fixed $\alpha \in \mathbb{R}^{m}, \alpha \neq 0$,

$$
\Omega(\alpha):=\{x \in \mathbb{G}:\langle\alpha, \pi(x)\rangle>0\},
$$

is a half-space to which our Liouville-type Theorem 1.1 applies. 
Example 1.4. In $\mathbb{R}^{N}=\mathbb{R}^{n} \times \mathbb{R}^{n} \times \mathbb{R}$, whose point is denoted by $(x, y, t), x, y \in \mathbb{R}^{n}$, $t \in \mathbb{R}$, consider the linear second order PDO

$$
\mathcal{L}=\Delta_{x}+\left(x \cdot \nabla_{y}-\partial_{t}\right)^{2} .
$$

This operator is a sub-Laplacian on a group $\mathbb{K}$ named in [6] of Kolmogorov-type. Taking into account the composition law and the dilations on $\mathbb{K}$ defined in 6 , Section 4.3.4], one immediately recognizes that the half-spaces to which our Liouvilletype Theorem 1.1 applies are of the kind

$$
\left\{(x, y, t) \in \mathbb{R}^{N}:\langle\alpha, x\rangle+\beta t>0\right\},
$$

where $|\alpha|^{2}+\beta^{2}>0$.

Our paper is organized as follows.

The next section is devoted to the notation, definitions, and results needed in the note.

In section 3 we will prove Theorem [1.1, Proposition 1.2, and Remark 3.1

\section{Sub-Laplacians AND Related SUb-haRmonic FUnCtions}

We call a sub-Laplacian on $\mathbb{R}^{N}$ any linear second order partial differential operator $\mathcal{L}$ of the kind

$$
\mathcal{L}=\sum_{j=1}^{m} X_{j}^{2}
$$

where the $X_{j}$ 's are smooth vector fields (i.e. linear partial differential operator of order one and smooth coefficients) satisfying the following conditions:

(H1) the Lie algebra

$$
a:=\operatorname{Lie}\left\{X_{1}, \ldots, X_{m}\right\}
$$

is a vector space of dimension $N$; moreover,

$$
\text { rank } a(x)=N \text { at any point } x \in \mathbb{R}^{N} \text {; }
$$

(H2) there exists a group of dilations $\left(\delta_{\lambda}\right)_{\lambda>0}$ in $\mathbb{R}^{N}$ such that every $X_{j}$ is $\delta_{\lambda^{-}}$ homogeneous of degree one.

A group of dilations in $\mathbb{R}^{N}$ is a family of diagonal linear functions $\left(\delta_{\lambda}\right)_{\lambda>0}$ of the kind

$$
\delta_{\lambda}\left(x_{1}, \ldots, x_{N}\right)=\left(\lambda^{\sigma_{1}} x_{1}, \ldots, \lambda^{\sigma_{N}} x_{N}\right), \quad x=\left(x_{1}, \ldots, x_{N}\right) \in \mathbb{R}^{N}
$$

where $\sigma_{1}=1 \leq \sigma_{2} \leq \cdots \leq \sigma_{N}, \sigma_{j} \in \mathbb{N}$.

Condition (H1) implies the hypoellipticity of $\mathcal{L}$ : in particular, the $\mathcal{L}$-harmonic functions, i.e., the solution to $\mathcal{L} u=0$, are smooth. Moreover, conditions (H1) and (H2) imply the existence of a group law $\circ$ in $\mathbb{R}^{N}$ such that $\mathbb{G}=\left(\mathbb{R}^{N}, \circ, \delta_{\lambda}\right)$ is a homogeneous Lie group on which the vector fields $X_{j}^{\prime} s$ are left translation invariant and $\delta_{\lambda}$-homogeneous of degree one (see [4]). The natural number

$$
Q=\sigma_{1}+\ldots+\sigma_{N}
$$

is called the homogeneous dimension of $\mathbb{G}$. Throughout the paper we always assume $Q \geq 3$ (if $Q=2$, then $\mathbb{G}$ is the Euclidean group). Then there exists a 
continuous function $d: \mathbb{G} \longrightarrow \mathbb{R}$, smooth and strictly positive outside the origin, $\delta_{\lambda}$-homogeneous of degree one and such that

$$
\gamma(x):=\left(\frac{1}{d(x)}\right)^{Q-2}
$$

is $\mathcal{L}$-harmonic in $\mathbb{R}^{N} \backslash\{0\}$ (see [6, Section 5.4]). This function $d$ is called an $\mathcal{L}$-gauge and for $\mathcal{L}$ plays a role analogous to the one played by the Euclidean norm with respect to the classical Laplacian. In particular, the $d$-balls

$$
B_{d}(x, r):=\left\{y \in \mathbb{G}: d\left(x^{-1} \circ y\right)<r\right\}
$$

support averaging operators characterizing the $\mathcal{L}$-harmonicity. To be precise, define

$$
\begin{gathered}
\psi:=\left|\nabla_{\mathcal{L}} d\right|^{2}, \quad \nabla_{\mathcal{L}}=\left(X_{1}, \ldots, X_{m}\right), \\
M_{r} u(x):=\frac{1}{c_{d} r^{Q}} \int_{B_{d}(x, r)} \psi\left(x^{-1} \circ y\right) u(y) d y
\end{gathered}
$$

and

$$
N_{r}(\mathcal{L} u)(x)=\frac{1}{(Q-2) c_{d} r^{Q}} \int_{0}^{r} \rho^{Q-1}\left(\int_{B_{d}(x, \rho)} \mathcal{L} u(y)\left(d\left(x^{-1} \circ y\right)^{2-Q}-\rho^{2-Q}\right) d y\right) d \rho
$$

where $c_{d}=\int_{B_{d}(0,1)} \psi d y$.

Then, if $\Omega$ is an open subset of $\mathbb{G}, u \in C^{2}(\Omega)$ and $\overline{B_{d}(x, r)} \subseteq \Omega$,

$$
u(x)=M_{r} u(x)-N_{r}(\mathcal{L} u)(x)
$$

(see [6, Theorem 5.6.1]).

We stress that $\psi$ is smooth outside the origin, $\delta_{\lambda}$-homogeneous of degree zero, and nonconstant unless $\mathbb{G}$ is the Euclidean group (see [5]; see also [6, Proposition 9.8.9]). In some particular important cases, such as, e.g., the group of Heisenberg type, explicit expressions of $\psi$ are known (see [6, Example 5.5.3]). In any case it is known that $\psi>0$ in a dense open subset of $\mathbb{R}^{N}$ (see [6, page 262]).

With these mean value operators, one can prove a version of the Gauss-Koebe Theorem in our setting (see [6, Section 5.6]):

Theorem 2.1 (Gauss-Koebe-type Theorem). If $\Omega \subseteq \mathbb{R}^{N}$ is open and $u: \Omega \longrightarrow \mathbb{R}$ is $\mathcal{L}$-harmonic, then

$$
u(x)=M_{r} u(x)
$$

for every $x \in \Omega$ and $r>0$ such that $\overline{B_{d}(x, r)} \subseteq \Omega$.

Vice versa, if $u$ is merely continuous in $\Omega$ and satisfies (2.2), then $u$ is $C^{\infty}$ and $\mathcal{L}$-harmonic in $\Omega$.

The average operator $M_{r}$ can also be used to fix the notion of $\mathcal{L}$-superharmonic function.

A lower semicontinuous function $u: \Omega \longrightarrow]-\infty, \infty]$ is called $\mathcal{L}$-superharmonic if $u$ is finite in a dense subset of $\Omega$ and

$$
u(x) \geq M_{r} u(x)
$$

for every $x \in \Omega$ and $r>0$ such that $\overline{B_{d}(x, r)} \subseteq \Omega$.

A quite exhaustive theory of $\mathcal{L}$-subharmonic functions is presented in the monograph [6, Chapter 8]. In particular, there it is proved that every $\mathcal{L}$-subharmonic 
function is $L_{\text {loc }}^{1}$ and that if $u$ is of class $C^{2}$, then $u$ is $\mathcal{L}$-subharmonic if and only if $\mathcal{L} u \geq 0$.

\section{Proof of Theorem 1.1, Proposition 1.2 and Remark 3.1}

The most important part of this section is the

Proof of Theorem 1.1. Let $\alpha \in \mathbb{E}, \alpha \neq 0$, be fixed and let

$$
\Omega(\alpha):=\{x \in \mathbb{G}:\langle\alpha, \pi(x)\rangle>0\} .
$$

For every $x \in \Omega(\alpha)$ we define

$$
r(x):=\varepsilon\langle\alpha, \pi(x)\rangle,
$$

where $\varepsilon>0$ is fixed in such a way that

$$
B(x, r(x)) \subseteq \Omega(\alpha) \quad \forall x \in \Omega(\alpha) .
$$

We will show in a moment the existence of a suitable $\varepsilon>0$ satisfying (3.1).

For a function $u \in L_{\text {loc }}^{1}(\Omega(\alpha))$ we let

$$
T(u): \Omega(\alpha) \longrightarrow \mathbb{R}, \quad T(u)(x):=M_{r(x)}(u)(x) .
$$

Hence,

$$
T(u)(x)=\int_{\Omega(\alpha)} K(x, y) u(y) d y, \quad x \in \Omega(\alpha)
$$

where

$$
K(x, y)=\frac{1}{c_{d}(r(x))^{Q}} \psi\left(x^{-1} \circ y\right) \mathcal{X}_{B_{x}}(y) .
$$

In what follows we also use the following notation:

$$
A_{x}:=\left\{y \in \Omega(\alpha) \mid d\left(y^{-1} \circ x\right)<r(y)\right\} .
$$

With this notation, we have

$$
\mathcal{X}_{B_{x}}(y)=\mathcal{X}_{A_{y}}(x)
$$

Indeed

$$
y \in B_{x} \Longleftrightarrow d\left(x^{-1} \circ y\right)<r(x) \Longleftrightarrow x \in A_{y} .
$$

Let us now show (3.1). We first remark that $\mathbb{E} \ni e \longmapsto d(e) \in \mathbb{R}$ is homogeneous of degree one with respect to the Euclidean dilation $e \longmapsto \lambda e$. As a consequence, by a suitable constant $c>0$, we have

$$
d(e) \geq c|e| \quad \forall e \in \mathbb{E}, \quad|\cdot|=\text { Euclidean norm. }
$$

Moreover, we can also assume that

$$
d(x) \geq c|\pi(x)| \quad \forall x \in \mathbb{G} .
$$

Then, if $x \in \Omega(\alpha)$, for every $z \in B_{d}(x, r(x))$, we have $r(x)>d(z, x) \geq c|\pi(z)-\pi(x)|$. Hence

$$
\begin{aligned}
\langle\alpha, \pi(z)\rangle & =\langle\alpha, \pi(x)\rangle+\langle\alpha, \pi(z)-\pi(x)\rangle \geq\langle\alpha, \pi(x)\rangle-|\alpha||\pi(z)-\pi(x)| \\
& \geq\langle\alpha, \pi(x)\rangle-\frac{|\alpha|}{c} r(x)=\langle\alpha, \pi(x)\rangle\left(1-\frac{|\alpha|}{c} \varepsilon\right) .
\end{aligned}
$$

Thus, if $0<\varepsilon<\frac{c}{|\alpha|}$, we get $\langle\alpha, \pi(z)\rangle>0$; i.e., $z \in \Omega(\alpha)$ and (3.1) is proved.

The proof of Theorem 1.1 will immediately follow from the next lemma. 


\section{Main Lemma.}

(i) $K(x, y) \geq 0$ for every $x, y \in \Omega(\alpha)$;

(ii) $\int_{\Omega(\alpha)} K(x, y) d y=1$ for every $x \in \Omega(\alpha)$;

(iii) $\int_{\Omega(\alpha)} K(x, y) d x=\int_{\Omega(\alpha)} K(x, \alpha) d x$ for every $y \in \Omega(\alpha)$;

(iv) $c^{*}:=\int_{\Omega(\alpha)} K(x, \alpha) d x>1$.

Proof of the Main Lemma.

(i) It straightforwardly follows from (3.2).

(ii) By the Gauss-Koebe-type Theorem 2.1 for $\mathcal{L}$-harmonic functions, if $u$ is $\mathcal{L}$-harmonic in $\Omega(\alpha)$, then $T(u)=u$. In particular $T(1)=1$, that is,

$$
1=\int_{\Omega(\alpha)} K(x, y) d y \text { for every } x \in \Omega(\alpha)
$$

(iii) This is the crucial part of the Main Lemma. We start by proving the following property of $\Omega(\alpha): \forall y \in \Omega(\alpha)$ there exists $\lambda=\lambda(y)>0$ such that

$$
\delta_{\lambda}(\alpha) \circ y^{-1} \circ x \in \Omega(\alpha) \text { and } r\left(\delta_{\lambda}(\alpha) \circ y^{-1} \circ x\right)=r(x)
$$

for every $x \in \Omega(\alpha)$.

Indeed, let $y, x \in \Omega(\alpha)$ and consider

$$
\begin{aligned}
\left\langle\alpha, \pi\left(\delta_{\lambda}(\alpha) \circ y^{-1} \circ x\right)\right\rangle & =\left\langle\alpha, \pi\left(\delta_{\lambda}(\alpha)\right)\right\rangle+\left\langle\alpha, \pi\left(y^{-1}\right)\right\rangle+\langle\alpha, \pi(x)\rangle \\
& =\langle\alpha, \pi(x)\rangle+\lambda\langle\alpha, \alpha\rangle-\langle\alpha, \pi(y)\rangle .
\end{aligned}
$$

Then, if we choose $\lambda=\frac{\langle\alpha, \pi(y)\rangle}{|\alpha|^{2}}$ we have $\lambda>0$ and

$$
\left\langle\alpha, \pi\left(\delta_{\lambda}(\alpha)\right) \circ y^{-1} \circ x\right\rangle>0, \quad r\left(\left(\delta_{\lambda}(\alpha) \circ y^{-1} \circ x\right)\right)=r(x) .
$$

This completes the proof of the stated property of $\Omega(\alpha)$.

In what follows we also use a homogeneity property of $x \longmapsto r(x)$, precisely

$$
r\left(\delta_{\lambda}(x)\right)=\lambda r(x) \text { for every } x \in \Omega(\alpha) \text { and } \lambda>0 .
$$

Indeed

$$
r\left(\delta_{\lambda}(x)\right)=\varepsilon\left\langle\alpha, \pi\left(\delta_{\lambda}(x)\right)\right\rangle=\varepsilon\langle\alpha, \lambda \pi(x)\rangle=\lambda r(x) .
$$


Let us now fix $y \in \Omega(\alpha)$ and compute

$$
\begin{aligned}
\int_{\Omega(\alpha)} K(x, \alpha) d x= & \frac{1}{c_{d}} \int_{\Omega(\alpha)}\left(\frac{1}{r(x)}\right)^{Q} \psi\left(x^{-1} \circ \alpha\right) \mathcal{X}_{B_{x}}(\alpha) d x \\
& \left(\operatorname{letting} \hat{\psi}(z)=\psi\left(z^{-1}\right)\right) \\
& =\frac{1}{c_{d}} \int_{A_{\alpha}}\left(\frac{1}{r(x)}\right)^{Q} \hat{\psi}\left(\alpha^{-1} \circ x\right) \mathcal{X}_{A_{\alpha}}(x) d x
\end{aligned}
$$

(using the change of variables $x=\delta_{\frac{1}{\lambda}}(\xi)$ and noticing that $r\left(\delta_{\frac{1}{\lambda}}(\xi)\right)=\frac{1}{\lambda} r(\xi)$ and that $\left.d x=\lambda^{-Q} d \xi\right)$$$
=\frac{1}{c_{d}} \int_{\delta_{\lambda}\left(A_{\alpha}\right)}\left(\frac{1}{r(\xi)}\right)^{-Q} \hat{\psi}\left(\alpha^{-1} \circ \delta_{\frac{1}{\lambda}}(\xi)\right) d \xi
$$

(keeping in mind that $\hat{\psi}$ is $\delta_{\lambda}$-homogeneous of degree zero)

$$
=\frac{1}{c_{d}} \int_{\delta_{\lambda}\left(A_{\alpha}\right)}\left(\frac{1}{r(\xi)}\right)^{Q} \psi^{-1}\left(\delta_{\lambda}\left(\alpha^{-1}\right) \circ \xi\right) d \xi
$$

We now choose $\lambda=\lambda(y)>0$ such that $r\left(\delta_{\lambda}(\alpha) \circ y^{-1} \circ x\right)=r(x)$ for every $x \in \Omega(\alpha)$ and use the change of variable

$$
\xi=\delta_{\lambda}(\alpha) \circ y^{-1} \circ x .
$$

We obtain

$$
\int_{\Omega(\alpha)} K(x, \alpha) d x=\frac{1}{c_{d}} \int_{y \circ \delta_{\lambda}\left(\alpha^{-1}\right) \circ \delta_{\lambda}\left(A_{\alpha}\right)}\left(\frac{1}{r(x)}\right)^{Q} \hat{\psi}^{-1}\left(y^{-1} \circ x\right) d x .
$$

On the other hand, as we will recognize in a moment,

$$
y \circ \delta_{\lambda}\left(\alpha^{-1}\right) \circ \delta_{\lambda}\left(A_{\alpha}\right)=A_{y}
$$

Then

$$
\int_{\Omega(\alpha)} K(x, \alpha) d x=\frac{1}{c_{d}} \int_{A_{y}}\left(\frac{1}{r(x)}\right)^{Q} \hat{\psi}\left(y^{-1} \circ x\right) d x=\int_{\Omega(\alpha)} K(x, y) d x
$$

and (iii) is proved.

We are left to prove (3.3). One has

$$
\begin{aligned}
x \in y \circ \delta_{\lambda}\left(\alpha^{-1}\right) \circ \delta_{\lambda}\left(A_{\alpha}\right) & \Longleftrightarrow z:=\alpha \circ \delta_{\frac{1}{\lambda}}\left(y^{-1} \circ x\right) \in A_{\alpha} \\
& \Longleftrightarrow d(z, \alpha)<r(z) .
\end{aligned}
$$

We know that $r(z)=\frac{1}{\lambda} r\left(\delta_{\lambda}(\alpha) \circ y^{-1} \circ x\right)=\frac{1}{\lambda} r(x)$, while

$$
d(z, \alpha)=d\left(\alpha^{-1} \circ z\right)=d\left(\delta_{\frac{1}{\lambda}}\left(y^{-1} \circ x\right)\right)=\frac{1}{\lambda} d\left(y^{-1}, x\right) .
$$

We have thus proved that

$$
x \in y \circ \delta_{\lambda}\left(\alpha^{-1}\right) \circ \delta_{\lambda}\left(A_{\alpha}\right) \Longleftrightarrow d\left(y^{-1} \circ x\right)<r(x) \Longleftrightarrow x \in A_{y} .
$$

This completes the proof of (iii).

(iv) Let $x_{0} \in \mathbb{G} \backslash \overline{\Omega(\alpha)}$ and consider the function

$$
v: \Omega(\alpha) \longrightarrow \mathbb{R}, \quad v(x)=\left(d\left(x_{0}^{-1} \circ x\right)\right)^{-1-Q} .
$$


Obviously the function $v$ is smooth in $\Omega(\alpha)$. Moreover, $v>0$ and $v \in$ $L^{1}(\Omega(\alpha))$. By using the left invariance of $\mathcal{L}$ on $\mathbb{G}$ and the form of $\mathcal{L}$ for radial functions 1 we also have

$$
\begin{aligned}
(\mathcal{L} v)(x) & =\left(\mathcal{L} d^{-Q-1}\right)\left(x_{0}^{-1} \circ x\right) \\
& =\psi\left(x_{0}^{-1} \circ x\right)((Q+1)(Q+2)-(Q+1)(Q-1)) d^{-Q-3}\left(x_{0}^{-1} \circ x\right) \\
& =3(Q+1)\left(\psi d^{-Q-3}\right)\left(x_{0}^{-1} \circ x\right) .
\end{aligned}
$$

Then $\mathcal{L} v>0$ in a dense open set of $\Omega(\alpha)$. As a consequence, using the representation formula (2.1), we get

$$
T(v)(x)-v(x)>0 \quad \forall x \in \Omega(\alpha),
$$

that is, $T(v)>v$ in $\Omega(\alpha)$. It follows that

$$
\begin{aligned}
\int_{\Omega(\alpha)} v d x & <\int_{\Omega(\alpha)} T(v) d x=\int_{\Omega(\alpha)}\left(\int_{\Omega(\alpha)} K(x, y) v(y) d y\right) d x \\
& =\int_{\Omega(\alpha)} v(y)\left(\int_{\Omega(\alpha)} K(x, y) d x\right) d y=c^{*} \int_{\Omega(\alpha)} v(y) d y .
\end{aligned}
$$

Then

$$
\int_{\Omega(\alpha)} v d x<c^{*} \int_{\Omega(\alpha)} v d y
$$

which implies $c^{*}>1$, since $\int_{\Omega(\alpha)} v d x>0$. This completes the proof of the Main Lemma.

We can now conclude the proof of Theorem 1.1. Since $u$ is $\mathcal{L}$-superharmonic, we have $T(u) \leq u$ in $\Omega(\alpha)$. Therefore,

$$
\begin{aligned}
\int_{\Omega(\alpha)} u d x \geq & \int_{\Omega(\alpha)} T(u) d x \\
& (\text { as in the proof of the Main Lemma (iv) }) \\
= & c^{*} \int_{\Omega(\alpha)} u d x .
\end{aligned}
$$

Then, since $c^{*}>1$,

$$
\int_{\Omega(\alpha)} u d x \leq 0
$$

which implies $u \equiv 0$ since $u \geq 0$ and lower semicontinuous.

Proof of Proposition 1.2. Let $d$ be a gauge function for $\mathcal{L}$ and define

$$
u(x)=\left(d\left(x_{0}{ }^{-1} \circ x\right)\right)^{-Q+2}, \quad x \in \Omega(\alpha),
$$

where, as before, $x_{0} \notin \overline{\Omega(\alpha)}$. The function $u$ is smooth in $\Omega(\alpha)$ and

$$
\mathcal{L}(u)(x)=\left(\mathcal{L} d^{2-Q}\right)\left(x_{0}{ }^{-1} \circ x\right)=0, \quad x \in \Omega(\alpha) .
$$

Moreover, $u>0$ and $u \in L^{p}(\Omega(\alpha))$ since, from the assumption $\frac{Q}{2}>\frac{p}{p-1}$, it follows that

$$
p(Q-2)>Q
$$

\footnotetext{
${ }^{1}$ If $w=f(d)$, then $\mathcal{L}(w)=\psi\left(f^{\prime \prime}(d)+\frac{Q-1}{d} f^{\prime}(d)\right)$ (see [6 Proposition 5.4.3]).
} 
Remark 3.1. The assumption $u \geq 0$ in Theorem 1.1 cannot be removed.

Indeed, if $x_{0} \notin \overline{\Omega(\alpha)}$, the function

$$
u_{k}(x):=\partial_{x_{N}}^{k}\left(d\left(x_{0}^{-1} \circ x\right)\right)^{2-Q}
$$

is $\mathcal{L}$-harmonic in $\Omega(\alpha)$ for every $k \in \mathbb{N}$, and $\delta_{\lambda}$-homogeneous of degree $2-Q-k \sigma_{N}$.

Then, if $k>\frac{2}{\sigma_{N}}, u_{k} \in L^{1}(\Omega(\alpha))$. Thus, with this choice of $k, u_{k}$ is a summable $\mathcal{L}$-harmonic function in $\Omega(\alpha)$ and $u_{k} \neq \equiv 0$.

We would like to stress that in the previous argument we used the following properties:

(i) the differential operator $\partial_{x_{N}}$ is $\delta_{\lambda}$-homogeneous of degree $\sigma_{N}$ and commutes with $\mathcal{L}$;

(ii) $\mathcal{L}$ is left translation invariant with respect to the composition law o;

(iii) $d^{2-Q}$ is $\mathcal{L}$-harmonic out of the origin.

\section{ACKNOWLEDGMENTS}

The author is deeply indebted to Ermanno Lanconelli for suggesting the problem and for bringing attention to the paper 14. Moreover, the author would like to thank the referees, whose criticism led the author to improve the first version of the paper.

\section{REFERENCES}

[1] Isabeau Birindelli and Alessandra Cutrì, A semi-linear problem for the Heisenberg Laplacian, Rend. Sem. Mat. Univ. Padova 94 (1995), 137-153. MR1370909 (97b:35027)

[2] I. Birindelli, I. Capuzzo Dolcetta, and A. Cutrì, Liouville theorems for semilinear equations on the Heisenberg group (English, with English and French summaries), Ann. Inst. H. Poincaré Anal. Non Linéaire 14 (1997), no. 3, 295-308, DOI 10.1016/S0294-1449(97)801382. MR1450950 (98d:35049)

[3] I. Birindelli and J. Prajapat, Nonlinear Liouville theorems in the Heisenberg group via the moving plane method, Comm. Partial Differential Equations 24 (1999), no. 9-10, 1875-1890, DOI 10.1080/03605309908821485. MR1708111 (2000k:35055)

[4] A. Bonfil'oli and È. Lankonelli, On left-invariant Hörmander operators in $\mathbb{R}^{N}$ : applications to the Kolmogorov-Fokker-Planck equations (Russian, with Russian summary), Sovrem. Mat. Fundam. Napravl. 36 (2010), 24-35, DOI 10.1007/s10958-010-0123-7; English transl., J. Math. Sci. (N. Y.) 171 (2010), no. 1, 22-33. MR2752647 (2012b:35038)

[5] Andrea Bonfiglioli and Ermanno Lanconelli, Gauge functions, eikonal equations and Bôcher's theorem on stratified Lie groups, Calc. Var. Partial Differential Equations 30 (2007), no. 3, 277-291, DOI 10.1007/s00526-006-0087-0. MR2332415 (2008j:43004)

[6] A. Bonfiglioli, E. Lanconelli, and F. Uguzzoni, Stratified Lie groups and potential theory for their sub-Laplacians, Springer Monographs in Mathematics, Springer, Berlin, 2007. MR:2363343(2009m:22012)

[7] A. Bonfiglioli and F. Uguzzoni, Nonlinear Liouville theorems for some critical problems on H-type groups, J. Funct. Anal. 207 (2004), no. 1, 161-215, DOI 10.1016/S0022-1236(03)001381. MR2027639 (2004k:35057)

[8] Lorenzo D'Ambrosio and Enzo Mitidieri, A priori estimates, positivity results, and nonexistence theorems for quasilinear degenerate elliptic inequalities, Adv. Math. 224 (2010), no. 3, 967-1020, DOI 10.1016/j.aim.2009.12.017. MR2628800(2011e:35109)

[9] Lorenzo D'Ambrosio and Enzo Mitidieri, A priori estimates and reduction principles for quasilinear elliptic problems and applications, Adv. Differential Equations 17 (2012), no. 910, 935-1000. MR2985680

[10] Alessia Elisabetta Kogoj and Ermanno Lanconelli, One-side Liouville theorems for a class of hypoelliptic ultraparabolic equations, Geometric analysis of PDE and several complex variables, Contemp. Math., vol. 368, Amer. Math. Soc., Providence, RI, 2005, pp. 305-312, DOI 10.1090/conm/368/06786. MR2126477 (2005j:35025) 
[11] Alessia E. Kogoj and Ermanno Lanconelli, Liouville theorems in halfspaces for parabolic hypoelliptic equations, Ric. Mat. 55 (2006), no. 2, 267-282, DOI 10.1007/s11587-006-0015-9. MR2279425 (2007h:35032)

[12] Alessia Elisabetta Kogoj and Ermanno Lanconelli, Liouville theorems for a class of linear second-order operators with nonnegative characteristic form, Bound. Value Probl. (2007), Art. ID 48232, 16. MR2291929 (2007k:35083)

[13] Alessia Elisabetta Kogoj and Ermanno Lanconelli, Liouville theorem for X-elliptic operators, Nonlinear Anal. 70 (2009), no. 8, 2974-2985, DOI 10.1016/j.na.2008.12.029. MR2509383 (2010e:35055)

[14] Francesco Uguzzoni, A Liouville-type theorem on halfspaces for the Kohn Laplacian, Proc. Amer. Math. Soc. 127 (1999), no. 1, 117-123, DOI 10.1090/S0002-9939-99-04519-0. MR $1458268(99 \mathrm{~b}: 35026)$

Basque Center for Applied Mathematics (BCAM), Mazzaredo, 14, E48009 Bilbao, Basque Country, Spain

Current address: Dipartimento di Matematica, Alma Mater Studiorum - Università di Bologna

Piazza di Porta, S. Donato, 5, 40126 Bologna, Italy

E-mail address: alessia.kogoj@unibo.it 